\title{
Kernos
}

Revue internationale et pluridisciplinaire de religion grecque antique

3| 1990

Varia

\section{Chronique des rencontres scientifiques et des expositions}

André Motte

\section{(2) OpenEdition}

Journals

Édition électronique

URL : http://journals.openedition.org/kernos/1010

DOI : 10.4000/kernos. 1010

ISSN : 2034-7871

Éditeur

Centre international d'étude de la religion grecque antique

Édition imprimée

Date de publication : 1 janvier 1990

ISSN : 0776-3824

Référence électronique

André Motte, "Chronique des rencontres scientifiques et des expositions », Kernos [En ligne], 3| 1990, mis en ligne le 19 avril 2011, consulté le 16 septembre 2020. URL : http://journals.openedition.org/ kernos/1010 


\title{
CHRONIQUE DES RENCONTRES SCIENTIFIQUES ET DES EXPOSITIONS
}

\author{
par André MOTTE
}

De manière à pouvoir informer à temps les lecteurs de la revue, la Rédaction souhaite être avertie, aussitôt que possible, des manifestations projetées; elle se fera un plaisir de les annoncer dans cette rubrique.

\section{A. Rencontres scientifiques}

\section{Rencontres récentes}

Du 8 au 10 mars 1989 s'est tenu, à l'Université libre de Bruxelles, le 1er Congrès européen de Philosophie organisé par le Centre de philosophie ancienne de cette Université et par la Société belge de Philosophie, sur le thème Herméneutique et nihilisme. Parmi les douze conférences présentées, deux sont propres à intéresser les spécialistes de l'Antiquité : H.G. GADAMER, La conscience herméneutique, condition de la compréhension de la pensée grecque, et M. RICHIR, Pour une herméneutique phénoménologique de la pensée mythologique. Des Actes sont prévus. Organisation : Prof. L. COULOUBARITSIS et R. LEGROS, Société belge de Philosophie, 143, av. Buyl, B - 1050 BRUXELLES.

Du 16 au 18 mars 1989 s'est tenu, à Liège, le IIe Colloque international organisé par le Centre d'Étude de la Religion Grecque Antique (Athènes) sur le thème Oracles et mantique en Grèce ancienne. Cette rencontre, présidée par le Professeur E. MOUTSOPOULOS, a réuni plus de cent participants représentant une quarantaine d'institutions. Trente conférences et communications y ont été présentées et ont été suivies de discussions. Plusieurs manifestations parallèles étaient organisées dans le cadre du colloque : une exposition sur les sanctuaires oraculaires de Grèce (voir "Chronique des expositions»), une séance d'hommage à Marie Delcourt, avec une exposition de ses œuvres (voir la rubrique "Personalia"), l'inauguration de la banque de données bibliographiques «Mentor» pour les publications parues entre 1945 et 1985 (voir rubrique ci-après). Les Actes du colloque sont publiés dans le présent 
volume de Kernos. Organisation : Prof. A. MOTTE et R. LAFFINEUR, 32, place du XX-Août, B - 4000 LIÈGE.

Du 16 au 18 mars 1989 s'est tenu, à l'Université de Lille III, un Congrès international sur le thème Sciences historiques, Sciences du passé et nouvelles technologies d'information. Bilan et évaluation. L'Antiquité classique en constituait le champ privilégié de référence et les matières suivantes ont été couvertes : linguistique, archéologie, papyrologie, épigraphie, numismatique, information scientifique et technique. Dans la quarantaine de communications présentées, une concernait tout spécialement la religion grecque : S. COLE (Illinois University, Chicago), Data Bases for the Study of Greek Religion. Des pré-actes ont été diffusés. Organisation : Prof. G. LOSFELD, Credo, Université de Lille III, B.P. 149, F - 59653 VILLENEUVE D'ASCQ Cédex.

Du 14 au 16 avril 1989 s'est tenu, à l'Université de Genève, un colloque international sur le thème L'Orphisme et Orphée. Quinze communications y ont été présentées : L. BRISSON, Damascius et l'Orphisme; C. CALAME, Éros initiatique et la cosmogonie orphique; J. BOTTÉRO, L'anthropogonie mésopotamienne et l'élément divin en l'homme; G. CASADIO, Métempsycose entre Orphisme et Pythagorisme; J. REDFIELD, The politics of Immortality; J. VINOGRADOV, Zur sachlichen und geschichtlichen Deutung der Orphiker-Plättchen von Olbia; D. SABBATUCCI, L'Orpheo attestato da Pausania; Ph. BORGEAUD, Rhésos et Arganthoné; M. SCHMIDT, Images d'Orphée; J. DOERIG, La tête qui chante; J. BREMMER, Orpheus : Wife, Gender, Age, Mysteries; A. MONNIER, L'Orphée des ethnologues; M. TORTORELLI GHIDINI, Euphrone : una divinità orfica; F. GRAF, Textes orphiques et rituel orphique; J. RUDHARDT, Quelques réflexions à propos des hymnes orphiques. Des Actes sont prévus. Organisation : Prof. Ph. BORGEAUD, Département des Sciences de l'Antiquité, Univ. de Genève, CH - 1211 GENÈVE 4.

Du 29 au 31 mai 1989 s'est tenu, à la Vrije Universiteit de Bruxelles et à l'Université de Liège, le premier colloque international du Groupe de contact interuniversitaire d'études chypriotes sur le thème Cypriote Terracottas. Quelques communications avaient trait à la religion : E. GORING et E. PELTENBURG, Terracotta Figurines and Ritual at 
Kissonerga-Mosphilia; J. BRETON CONNELLY, Continuity and Change: the Cypriote Votive Tradition and the Hellenistic koinè; A. QUEYREL, Calathoi en terre cuite à décor de sphinx; R. LAFFINEUR, La bijouterie chypriote d'après le témoignage des terres cuites : l'exemple des statuettes d'Arsos (à propos des statues de culte). Le colloque s'est terminé par une journée de travail à Amsterdam, à l'occasion de l'inauguration de l'exposition Cyprus and Aphrodite, présentée à l'Allard Pierson Museum. Les actes de cette réunion seront édités en 1990 par Robert LAFFINEUR et Frieda VANDENABEELE.

Du ler au 6 septembre 1989 s'est tenu, à Pérouse, le IIe Symposium Platonicum consacré au Phèdre de Platon. Cette rencontre a réuni quelque cent vingt participants; huit conférences en sessions plénières et plus de trente communications ont été présentées, dont trois abordaient directement des thèmes religieux : $\mathrm{R}$. BODÉÜS, Les éléments d'une philosophie des dieux dans le Phèdre; C. EGGERS LAN, Zeus y alma del mundo en el Fedro, 246e-253c; A. MOTTE, L'aventure spirituelle du Phèdre et la prière. Des Actes sont prévus. Organisation : Prof. L. ROSSETTI, Istituto di Filosofia, Università di Perugia, Piazza G. Ermini, I - 06100 PERUGIA PG.

À la faveur de ce congrès a été créée une Société platonicienne internationale; son président, C.J. ROWE, a été chargé d'organiser le IIIe Symposium qui aura lieu à Bristol, en 1992, et aura pour thème le Politique.

Du 14 au 16 septembre 1989 s'est tenu, à Liège, un colloque international organisé par le Centre de recherches mythologiques de Paris $X$ Nanterre et le Centre d'histoire des religions de l'Université de Liège sur le thème Mythe et politique. Dans la vingtaine de conférences et de communications présentées, un nombre important concernait les mythes antiques et leurs survivances : A. BILLAULT, Un sage en politique : Apollonios de Tyane et les empereurs romains; G. CAPDEVILLE, Servius Tullius et le mythe du premier roi; L. COUlOUBARITSIS, Le statut du mythe de Gygès chez Platon; B. DEFORGE, Le mythe des Sept contre Thèbes; J. DESAUTELS, De l'ambiguïté $d u$ mythe; C. GARCÍA GUAL, La modération attique de Thésée; F. JOUAN, Les Corinthiens en Acarnanie et leurs prédécesseurs mythiques; H. MATHIEU, Les mythes des sociétés de femmes et leurs interprétations politiques; A. MOREAU, Le "retour des cendres» : Oreste et Thésée, deux cadavres (ou deux mythes) au service de la propagande 
politique; M. PETYCHAKIS, Les fonctions sociales des mythes politiques; Y. VERNIÈRE, Le voyage mythique d'Osiris-Dionysos en Asie et ses prolongements politiques; P. WATHELET, Le mythe d'Énée dans l'épopée homérique. Sa survie et son exploitation politique. Les Actes paraîtront dans la Bibliothèque de la Faculté de philosophie et lettres de l'Université de Liège. Organisation : Prof. Fr. JOUAN, Univ. de Paris X, F- 92001 NANTERRE Cédex, et A. MOTTE, Univ. de Liège, 32, place du XX-Août, B - 4000 LIÈGE.

Du 15 au 16 septembre 1989 s'est tenu, à l'Academia Belgica de Rome, dans le cadre de la célébration du cinquantenaire de cette institution, une Table ronde sur le thème Héraclès, d'une rive à l'autre de la Méditerranée. Bilan et perspectives. Les communications suivantes ont été présentées : F. BADER, Héraclès et l'idéologie tripartie; C. BAURAIN, Héraclès dans l'épopée homérique; C. BONNET, Héraclès en Orient : interprétations et syncrétismes; W. BURKERT, Eracle e gli altri eroi culturali del Vicino Oriente; A. HERMARY, L'influence procheorientale dans l'élaboration de l'iconographie d'Héraclès; M. JOST, Héraclès en Arcadie; C. JOURDAIN-ANNEQUIN, Héraclès en Occident; M. LE GLAY, Héraclès-Hercule en Afrique du Nord; M. PIÉRART, Les honneurs de Persée et d'Héraclès; D. SABBATUCCI, Ercole e la fondazione del culto dell' Ara Massima; F. VAN WONTERGHEM, Il culto di Ercole tra $i$ popoli oscosabellici. Des Actes sont prévus. Organisation : C. BONNET, Facultés Universitaires Notre-Dame de la Paix, Dpt d'histoire, 61, rue de Bruxelles, B - 5000 NAMUR, et C. JOURDANANNEQUIN, Université des Sciences Sociales de Grenoble II, U.E.R. Histoire et histoire des Arts, B.P. 25X, F - 38040 GRENOBLE.

Du 24 au 27 septembre 1989 s'est tenu, à Bruxelles et à Liège, le 1er Colloque E. Lamotte sur le thème Histoire du Boudhisme. Organisation: Institut orientaliste de l'Université Catholique de Louvain-la-Neuve et Section d'Histoire et de Philologie Orientales de l'Université de Liège (secrétariat du colloque), 32, place du XX-Août, B 4000 LIËGE.

Du 3 au 5 novembre 1989 s'est tenu, à Comacchio, un colloque sur le thème Dionysos. Mito e Mistero. Les communications suivantes ont été présentées : P.E. ARIAS, Avviamento ad una lettura su Dionysos; 
C. BÉRARD, Dionysos : la maschera impossibile; G. BERMOND MONTANARI, Dionysos sui vasi attici a figure nere rinvenuti nei recenti scavi dei Giardini Margherita a Bologna; F. BERTI-D. BALDONI, Documenti del culto di Dionysos a Spina : le choes; M. BONAMICI, Una nuova rappresentazione di Dionysos in Etruria; A. BOTTINI, Appunti sulla presenza di Dionysos nel mondo italico; G. CASADIO, Dionysos e Semele : morte di uno dio e resurrezione di una donna; $\mathrm{O}$. DE CAZANOVE, Theòs en askò : osservazioni sui meccanismi di trasmissione della figura di Dionysos; I. CHIRASSI COLOMBO, Dionysos-Bacchos e la città estatica : immagini, messaggi e modelli di buon disordine; G. COLONNA, Tracce di "dionisismo con Dionysos», nell' Etruria preellenistica; E. CORRADINI, La fortuna di Dionysos nel collezionismo antico e moderno; G. DORFLES, L'estetica del mito; F. FARINA, $I l$ dionisiaco nell arte contemporanea; F. FRONTISI DUCROUX, La maschera del dio e il dio maschera; O.E. GHIANDONI, $L a$ lekane della tomba 268A di Valle Pega, a Spina : un ulteriore contributo alle scene di ierogamia di Dionysos e Ariadne; F. GILotTA, Aspetti dionisiaci su alcuni documenti di pittura vascolare spinetica; G. GUALANDI, Le raffigurazioni del dio Dionysos in Grecia e in Etruria padana; E. HOSTETTER, Il carattere del servizio bronzeo del simposio in alcune tombe di Spina; C. ISLER KERÉNYI, Dionysos : dio delle donne; F. LISSARRAGUE, Le figure del corteggio dionisiaco; F. LOPERFIDO, Dionysos e la categoria del divino fanciullo; A. MASTROCINQUE, Giganti silenici in Grecia ed Etruria; V. OTTOLENGHI, La danza : tra Dionysos ed Apollo; A. PRIVITERA, La nascita della tragedia : il ruolo del ditirambo; F. RAUSA, Dionysos nella Gigantomachia raffigurazioni del mito su alcuni vasi di Spina; D. RESTANI, Dionysos tra kythara ed aulos : un percorso di iconografia musicale; $\mathrm{D}$. SABBATUCCI, Dionysos figura mitica e divinità orgiastica; P. SCARPI, Geografie mitiche di Dionysos; L. TONDO, Riflessi numismatici della vittoria di Dionysos; F.M. VANNI, Aspetti dell' iconografia di Dionysos nella monetazione di Leptis Magna. Des Actes sont prévus et peuvent être déjà commandés. Secrétariat : Museo Archeologico Nazionale, Via XX Settembre, 124, I - FERRARA.

Du 13 au 17 novembre 1989 s'est tenue, à l'Université de Valladolid, une session de cours, suivis de discussions, sur le thème Introduccion a la Religión Griega. Le programme comprenait : J. ALSINA CLOTA, Religión y literatura; J. GARCIA LOPEZ, Caracteristicas Generales de la Religión Griega; C. GARCIA GUAL, Religión y mito; M. GARCIA TEIJEIRO, Religión y magia; A. MOTTE, Religion et Philosophie : le même et l'autre; A. PEREZ JIMENEZ, Religión y Politica. Organisation : 
Prof. E. SUAREZ DE LA TORRE, Facultad de Filosofia y Letras, Departamento de Filologia Clásica, E - 47002 VALLADOLID.

Du 15 au 18 novembre s'est tenu, à Liège, sur le thème Phoinikeia Grammata (Function and Diffusion of Writing in the Ancient Mediterranean) le IXe colloque organisé par le Groupe de contact interuniversitaire d'études phéniciennes et puniques. Une cinquantaine de communications ont été présentées. Les Actes paraîtront dans la collection Studia Phonicia, XII. Organisation : Prof. C. BAURAIN, Université de Liège, Séminaire d'histoire grecque, 32, place du XX-Août, B - 4000 LIÈGE.

Du 23 au 25 novembre 1989 s'est tenu, à Naples, un colloque international sur le thème Les bois sacrés, organisé par le Centre J. Bérard et $l^{\prime}$ École Pratique des Hautes Études, Ve Section. Communications de M. BEARD, C. FRONTISI, M. CARTRY, F. GRAF, CH. JACOBS, F. ZEVI, F. COARELli, J.-L. BRUNAUX, M. DETIENNE, C. MONTEPAONE, D. BRIQUEL, M. LEJEUNE, T.F.C. BLAGG, O. DE CAZANOVE, G. CAPDEVILLE, H. BROISE, J. SCHEID, C. AMPOLO. La plupart des communications concernaient les domaines grec et romain. Secrétariat: Centre J. Bérard, 86, Via Francesco Crispi, I - 80121 NAPOLI.

Du 27 novembre au 1er décembre 1989 s'est tenue, à Rome, la VII Settimana di Studi organisée par le Centro Studi Sanguis Christi sur le thème Sangue et antropologia nella Teologia medievale. Le programme prévoyait plus de 60 communications dont plusieurs concernaient directement l'Antiquité, voire la religion grecque : B. ENRICO, $I l$ concetto di sangue presso gli Ittiti : reflessioni alla luce dei testi religioso-culturali et mitologici; G. CASADIO, Problemi di antropologia manichea; L. FURIANI, Tematiche del sangue nel romano greco d'amore; R. LAURENT, Spunti per uno studio di aima in Plutarco; C. SANTANIELLO, La flagellazione all' altare di Orthia : antichità e forma di rito spartano; M. SCARSI GARBUGINO, Il sangue e il fiore : Giacinto e Aiace. Organisation : Prof. F. VATTIONIONI, Via Narni, 29, I - 00181 ROMA. 
Durant la saison 1989-1990, l'Association Culturelle Belgohellénique "Alexandre le Grand» organise à Charleroi, un cycle de trois conférences sur le thème Mythe et religion : réalité de l'homme antique. Programme : P. MARCHETTI, La construction d'un temple : un acte sacré et ... mathématique; L. COULOUBARITSIS, Mythe et philosophie dans la pensée ancienne; A. MOTTE, Le message religieux d'Éleusis. Adresse de l'Association : 20, boulevard Tirou, bte 24, B - 6000 CHARLEROI.

\section{Rencontres annoncées}

Les 21 et 22 mars 1990 se tiendra, à Louvain-la-Neuve et à Liège, sur le thème Expérience religieuse et expérience esthétique, le Ve colloque interuniversitaire organisé par les Centres d'histoire des religions de ces deux Universités. Plusieurs communications concerneront l'Antiquité : O. CLÉMENT, L'icône, théologie de la beauté; L. COULOUBARITSIS, Le statut du symbole dans l'icône; A. HAQUIN, Originalité des rites chrétiens de sépulture aux premiers siècles; V. PIRENNE-DELFORGE, Beauté des dieux et beauté des hommes en Grèce ancienne; J. RIES, L'expérience de la lumière dans le manichéisme; P. SOMVILLE, Symbole et iconographie : "le voile marin de la déesse»; Ch.M. TERNES, L'aménagement des téménoi gallo-romains et le rituel.

La 3e Rencontre égéenne internationale de l'Université de Liège se tiendra les 23, 24 et 25 avril 1990 à la Station de recheches sous-marines et océanographiques de l'Université de Liège (Stareso), à Calvi, Corse, sur le thème Thalassa. L'Égée préhistorique et la mer. The Prehistoric Aegean and the Sea. Trente-cinq communications sont prévues, réparties en huit sections : 1 . Bateaux et navigation; 2. Installations portuaires; 3 . Fouilles subaquatiques; 4 . Contacts et relations commerciales; 5 . Le monde égéen, la Méditerranée et la Mer Noire; 6. Iconographie; 7. La mer et le mythe; 8. Sources écrites, cadre naturel.

Du 14 au 16 juin 1990, à l'occasion du 10e anniversaire de sa fondation, l'Association luxembourgeoise Pro Latinitate organise à Luxembourg, en collaboration avec le Centre universitaire du pays ainsi que les Universités de Liège et de Metz, un colloque sur le thème Parole sacrée, parole profane. De la religion à l'éloquence. Organisation : Mme R. KIEFFER, 10, rue Lemire, L - 1927 LUXEMBOURG. 
En août 1990 se tiendra, à Athènes et à Samos, le Deuxième Congrès International de philosophie grecque organisé par l'Association internationale de philosophie grecque sur le thème La philosophie de Socrate. Cinq groupes de travail sont prévus : Socrate et les Présocratiques; Socrate, Platon, les Sophistes et Aristote; Socrate, Aristophane et Xénophon; problèmes socratiques philosophiques; le portrait philosophique de Socrate. Organisation : Prof. Constantin BoUdOURIS, 5, rue Simonidou, GR - 17456 ALIMOS.

Du 20 août au 3 septembre 1990 se tiendra, à Paris et en Turquie, sur le thème Le héros, le IIe Congrès de mythologie et psychothérapie organisé par la Société de Recherches Psychothérapiques de langue française, par le Centre de Recherches Mythologiques de Paris XNanterre et le Centre de Recherches sur le symbolisme de la Mère de l'Université de Caen. Cinq tables rondes se tiendront à Paris, du 20 au 22 août, et trois en Asie Mineure, au cours du voyage (23 août au 3 septembre) qui comportera les étapes suivantes : Izmir, Éphèse, Antalya, Xanthos, Didyme, Milet, Priène, Sardes, Pergame, Troie, Istanbul. Thèmes des tables rondes : 1 . Les modes d'accomplissement du héros : naissance, initiation, conquête; 2 . Le héros protecteur, fondateur et miroir de la société; 3 . Le héros, archétype et moteur de l'évolution créatrice; 4 . Psychothérapie du héros : pulsions, angoisses et folies. Y at-il des médications pour l'héroïsme ?; 5 . Les substituts actuels du héros et de son image. Où sont les héros des temps modernes ?; 6. La place du héros entre l'humain et le divin; 7. Le héros et sa famille; 8 . La mort du héros. Y a-t-il mort de l'héroïsme?

Renseignements et propositions de communications : B. DEFORGE, 66, route de Bretagne, F - 14760 BRETTEVILLE / ODON, Tél. 31-73-49-67; F. JOUAN, 19, rue des Carmélites, F - 14300 CAEN, Tél. 31-86-17-89; Pr. A. MATHÉ, 42, avenue Mozart, F - 75016 PARIS, Tél. 42-25-29-49.

Inscriptions uniquement auprès du Pr. A. MATHÉ.

Du 3 au 9 septembre 1990 se tiendra, à Rome, le XVIIe congrès de l'Association Internationale pour l'Histoire des Religions (I.A.H.R.) sur le thème La notion de religion dans la recherche comparative. Une section concernera la Grèce et Rome. Les inscriptions pour les communications sont clôturées. Organisation : Prof. U. BIANCHI, 
Università di Roma «La Sapienza», 5, piazzale Aldo Moro, I - 00185 ROMA.

Du 24 au 28 septembre 1990 se tiendra, à Santiago du Chili, sur le thème Visión y Audición en el mundo clásico, la seconde Rencontre internationale organisée par le Centro des Estudios Clasicos. Organisation : G. GRAMMATICO AMARI, Universitad Metropolitano de Ciencias de la Educación, Av. J.-P. Alessandri, 774, SANTIAGO (CHILI).

Le colloque sur le thème L'initiation dans les cultures hellénique et romaine qu'organise le Séminaire d'études des mentalités religieuses (S.E.M.A.) de l'Université de Montpellier est reporté d'un an et aura lieu durant la dernière semaine d'avril 1991. Organisation : Prof. A. MOREAU, Univ. P. Valéry, B.P. 5043, F - 34032 MONTPELLIER Cédex.

\section{B. Expositions}

Sanctuaires oraculaires de Grèce (Liège, 16-31 mars 1989)

En complément au colloque Oracles et mantique en Grèce ancienne, une exposition a été organisée dans les locaux du siège régional de la Générale de Banque afin d'intéresser un plus large public à cette importante manifestation du sacré dans l'antiquité gréco-romaine. L'exposition rassemblait, sur une dizaine de panneaux, des photographies, des plans et des notices qui voulaient faire connaître quelques-uns des sites oraculaires les plus représentatifs et les mieux connus en évoquant leur origine, leur histoire, leur topographie, la divinité, le fonctionnement de l'oracle proprement dit : oracle de Zeus à Dodone, oracles d'Apollon à Delphes, Claros et Didymes, oracles d'Asclépios à Épidaure et Corinthe, oracle d'Amphiaraos à Oropos, sanctuaire d'Éphyra de Thesprotie.

L'ensemble a été préparé dans le cadre d'un cours de licence d'archéologie grecque à la Section d'histoire de l'art et archéologie de l'Université de Liège. Les étudiants ont pris une part importante à sa réalisation.

L'exposition a été aussi l'occasion de développer le thème du colloque dans le cadre de l'Égypte ancienne. Des panneaux réalisés par l'a.s.b.l. Egyptologica illustraient le sujet suivant trois axes : I. Pourquoi recourir à l'oracle ? La situation politique et religieuse; II. Les circonstances matérielles de l'oracle; III. L'oracle : un instrument de 
propagande politique. L'utilisation de l'oracle par le pouvoir politique à l'époque pharaonique, notamment sous Thoutmosis III et à la troisième Période intermédiaire, montre en quoi Alexandre le Grand s'est comporté tel un souverain égyptien en faisant reconnaître sa légitimité par le clergé d'Amon à Siwa.

Un poster était enfin consacré au thème de l'oracle, source d'inspiration des artistes contemporains, plus particulièrement à la gravure "'t Orakel» de Paul Joostens (1889-1960), représentant la façade du Trésor de Siphnos à Delphes.

Robert Laffineur

Université de Liège
Florence Doyen

a.s.b.l. Egyptologica

Au mêmes dates, une exposition des œuvres de Marie Delcourt (18911979) a aussi été organisée, à Liège, dans le cadre de ce colloque. La même exposition a été présentée aux Archives Nationales du Luxembourg durant la seconde quinzaine d'octobre 1989. Parmi les documents exposés figuraient les éditions successives des œuvres de M. Delcourt se rapportant à la religion et à la mythologie grecques. Organisation : Mlle I. MALAISE, Centre d'histoire des religions de l'Université de Liège, 32, place du XX-Août, B - 4000 LIÈGE.

Du 7 mai au 17 décembre 1989 s'est tenue, au Palazzo Bellini de Comacchio, une exposition sur Dionysos. Mito e Mistero. Les quelque cent objets sélectionnés, provenant des fouilles de la nécropole de Spina, illustraient les différents aspects du mythe de Dionysos dans le milieu grec et étrusque, du VI au IVe s. av. J.-C. Secrétariat : Museo Archeologico Nazionale, Via XX Settembre, 124, I - FERRARA.

Du 3 juin au 3 septembre s'est tenue, à Amsterdam, une exposition organisée par l'Archeologisch Museum der Universiteit van Amsterdam sur le thème Cyprus \& Aphrodite. Catalogue disponible : Oude Turfmarkt, 127, NL - AMSTERDAM. 